\title{
Isothermal Permeability of the Argillaceous Cobourg Limestone
}

\author{
A.P.S. Selvadurai* and M. Najari \\ Department of Civil Engineering and Applied Mechanics, McGill University, 817 Sherbrooke Street West, Montréal, QC, H3A OC3 - Canada \\ e-mail: patrick.selvadurai@mcgill.ca \\ * Corresponding author
}

\begin{abstract}
The paper presents the results of an experimental evaluation of the permeability of the heterogeneous argillaceous limestone from the Cobourg formation located in southern Ontario, Canada. The low permeability of the limestone necessitated the development of experimental techniques for effectively measuring the permeability of the rock. In particular, we examine the results of pressurization of a fluid-filled co-axial cylindrical cavity of finite length cored into a $150 \mathrm{~mm}$ diameter cylinder of the Cobourg Limestone. The orientation of the cavity is either normal to or along the nominal stratifications identified by the argillaceous partings separating the quartzitic phases. Both steady state and transient tests are used to estimate the permeability of the Cobourg Limestone. The paper also investigates the influence of a nominal axial load on the permeability of the rock.
\end{abstract}

Résumé - Perméabilité isotherme du Calcaire argileux de Cobourg — L'article présente les résultats d'une évaluation expérimentale de la perméabilité du calcaire argileux hétérogène de la formation de Cobourg, située dans le sud de l'Ontario au Canada. La faible perméabilité du calcaire a nécessité le développement de techniques expérimentales permettant de mesurer efficacement la perméabilité de la roche. En particulier, nous examinons les résultats de la pressurisation d'une cavité cylindrique coaxiale remplie de fluide et de longueur finie ancrée dans un cylindre de $150 \mathrm{~mm}$ de diamètre du Calcaire de Cobourg. L'orientation de la cavité est, soit perpendiculaire à, soit le long des stratifications nominales identifiées par les barres argileuses qui séparent les phases quartzitiques. Des tests transitoires à l'état stable sont utilisés pour estimer la perméabilité du Calcaire de Cobourg. L'article étudie également l'influence d'une charge axiale nominale sur la perméabilité de la roche.

\section{INTRODUCTION}

Permeability is a fundamental property of rocks and other geomaterials that characterizes the ability for fluids to permeate through the accessible saturated pore space under a hydraulic potential gradient. The range of permeabilities encountered in environmental geosciences and rock mechanics tends to vary from values for limestone: $\sim 10^{-14} \mathrm{~m}^{2}$ to $\sim 10^{-15} \mathrm{~m}^{2}$ (Selvadurai and Głowacki, 2008; Selvadurai and Selvadurai, 2010); concrete: $\sim 10^{-17} \mathrm{~m}^{2}$ to $\sim 10^{-20} \mathrm{~m}^{2}$ (Aldea et al., 1999; Mehta and Monteiro, 2014); cement grout: $\sim 10^{-20} \mathrm{~m}^{2}$ to $\sim 10^{-21} \mathrm{~m}^{2}$ (Selvadurai and Carnaffan, 1997); granite: $\sim 10^{-18} \mathrm{~m}^{2}$ to $\sim 10^{-20} \mathrm{~m}^{2}$ (Selvadurai et al., 2005; Selvadurai and Najari, 2013, 2015), Callovo-Oxfordian Argillite: $\sim 10^{-18} \mathrm{~m}^{2}$ to $\sim 10^{-21} \mathrm{~m}^{2}$ (Davy et al., 2007, 2009) to the Cobourg Limestone: $\sim 10^{-19} \mathrm{~m}^{2}$ to $\sim 10^{-22} \mathrm{~m}^{2}$ (Vilks and Miller, 2007; Selvadurai et al., 2011; Selvadurai and Jenner, 2012). There is no strict definition of what constitutes 
a low permeability material. Generally, rocks and other cementitious and synthetic materials with permeabilities less than $10^{-20} \mathrm{~m}^{2}$ are regarded as low permeability materials. Measuring the permeability of such material presents a challenge both from the experimental and modeling points of view. There is a school of thought that at such low permeabilities the processes of moisture movement within the pore space can range from Darcian flow resulting from a fluid pressure gradient to moisture diffusion resulting from a moisture concentration gradient with both processes present in the pore space satisfying the relevant mass and momentum conservation laws. While theoretical developments in these topics are extensive (Bear, 1972; Coussy, 1995; Selvadurai, 2015a) the combined treatment of both fluid flow and moisture diffusion within the same pore space will not be complete without a consideration of irreversible thermodynamic processes associated with the theory of multiphase mixtures (Green and Naghdi, 1970; Bowen, 1976) and references to these are given by Atkin and Craine (1976), Coussy (1995) and Selvadurai (2015a). In unsaturated regions, where moisture diffusion can exist, the diffusive processes can also be influenced by pore morphology (David, 1993), surface chemistry and surface tension effects. The application of such developments to engineering situations where the direct estimation of flow and leakage through porous media is straightforward in theory, but requires the development of an entirely new generation of experimental techniques to accurately determine the additional transport parameters with thermo-mechanical influences. Such all encompassing experimental techniques are currently unavailable. The prudent first approximation therefore is to examine fluid flow, even in low permeability materials, by considering the Darcian assumption of fluid transport through mechanically derived influences such as pressure gradients.

The work reported in this paper focuses on the permeability measurement of argillaceous rocks in the context of the deep geologic disposal of radioactive wastes. In general, most deep geologic disposal concepts for nuclear waste management also rely on engineered barriers that include the waste containers and engineered clay barriers (Selvadurai, 1996a, b, 2002; Selvadurai and Nguyen, 1997; Alonso et al., 2005; Gens, 2011). Nonetheless, it places a great deal of reliance on the repository rocks to act as natural geological barriers to attenuate, in the long term, radionuclide migration from the waste repository to the geosphere. The permeability of the geological formation that includes both intact and fractured regions is therefore of central importance to the feasibility of a deep geologic disposal concept (Pusch, 1990; Hueckel and Peano, 1996; Selvadurai and Nguyen, 1997; Selvadurai, 1997; Hoteit et al., 2002; Alonso and Gens, 2002; Alonso et al., 2005). In addition to the natural geological barriers, there is also considerable interest in developing low permeability cementitious materials that have a low shrinkage potential for use in other barrier systems including temporary sealing of excavation damage zones and fracture systems, bulkheads in galleries and seals for access shafts. Low permeability materials also feature prominently in concepts for very deep geologic disposal of hazardous wastes (Testa, 1994; Fuenkajorn and Daemen, 1996; Selvadurai, 2006). Similar sealing requirements are also necessary for re-habilitating abandoned wells that can be encountered in the geologic disposal of carbondioxide in fluidized forms (Pijaudier-Cabot and Pereira, 2013; Selvadurai, 2012, 2013).

Although granitic rocks have been considered by several countries for the construction of Deep Geologic Repositories (DGR) for long-term management of nuclear waste, attention is also given to argillaceous formations. Recent international examples include the Mesozoic Age Callovo-Oxfordian Formation in France, the Opalinus Clay in Switzerland and the non-indurated Boom Clay in Belgium (Mazurek, 2004; NWMO, 2011). Among others, key reasons for examining the characteristics of these argillaceous rocks include the ability of such rocks to accommodate deformations without extensive damage and rupture and their inherent self-sealing capacity that enables maintenance of low rock mass permeabilities (Horseman, 2001; Maßmann et al., 2009; Bock et al., 2010).

In Canada, a proposal exists for a Low and Intermediate Level radioactive Wastes (L\&ILW) DGR within a Paleozoic Age sedimentary sequence on the eastern flank of the Michigan Basin near Kincardine, Ontario (NWMO, 2011). Within the almost $840 \mathrm{~m}$ thick sedimentary sequence the repository would be positioned at a depth of approximately $680 \mathrm{~m}$ within the argillaceous Cobourg Limestone formation overlain by $200 \mathrm{~m}$ of illitic Ordovician Shales (NWMO, 2011; Joint Review Panel Report, 2015). Extensive studies conducted between 2006 and 2010 established the site specific bedrock stratigraphy and lateral traceability, the physical and chemical hydrogeological and geomechanical properties of the host and confining natural barrier formations. The suitability of the Cobourg Formation to host the proposed DGR is discussed by Mazurek (2004) and aspects related to the characterization of the mechanical properties of the Cobourg Limestone are given by Selvadurai (2015b). At the proposed location for the DGR, the geologic formations are near horizontally stratified (dip $0.6^{\circ}$ ) with the Cobourg Formation approximately $27 \mathrm{~m}$ thick and overlain by $200 \mathrm{~m}$ of upper Ordovician Age shales comprising the Queenston, Georgian Bay and Blue Mountain formations. This sequence is underlain by approximately $150 \mathrm{~m}$ of low permeability limestones and dolostones of the Black River and Trenton groups that rest on a Cambrian Sandstone and crystalline Pre-Cambrian basement. The stratigraphic sequence was determined from 8 deep cored boreholes and 299 historical oil and gas exploration well records at closest approach $3 \mathrm{~km}$ to the site. Reports by Golder Associates 

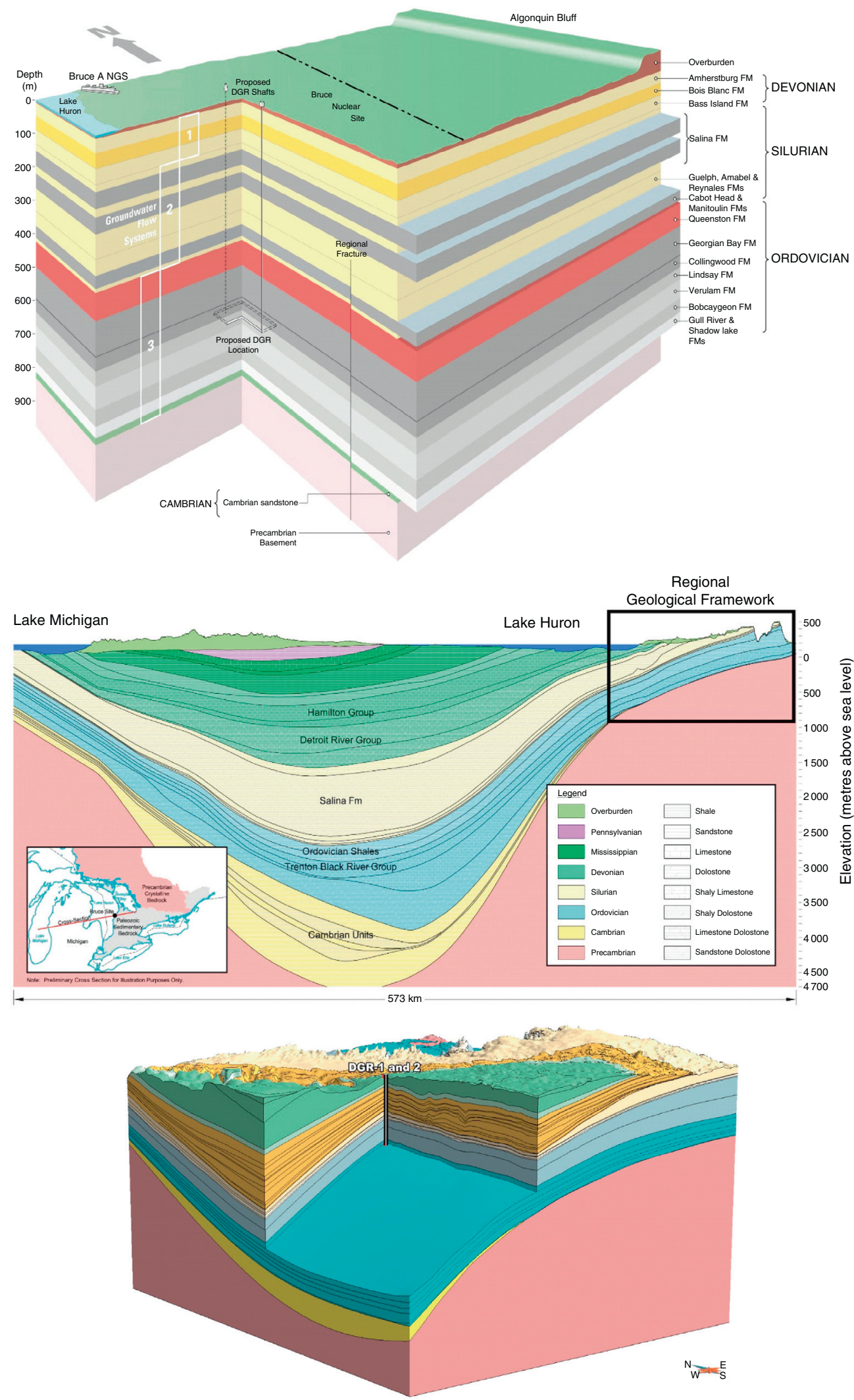

Figure 1

Regional geological framework and the location of the DGR beneath the Bruce site. (OPG, 2008). Approx. $40 \times$ vertical exaggeration. 
(2003), Mazurek (2004) and NWMO (2011) document the hydrogeological and geomechanical properties of the geologic strata encountered at the proposed L\&ILW deep geological repository site. Of particular interest to this research are the estimates for the hydraulic conductivity distribution with depth over the entire sequence of rocks, which range from $10^{-5} \mathrm{~m} / \mathrm{s}$ in the Devonian and upper Silurian near surface aquifers to between $10^{-11} \mathrm{~m} / \mathrm{s}$ to $10^{-15} \mathrm{~m} / \mathrm{s}$ in the Ordovician aquiclude proposed to host the DGR (NWMO, 2011; Beauheim et al., 2014) (Fig. 1). This translates to permeability of approximately $10^{-18} \mathrm{~m}^{2}$ to $10^{-22} \mathrm{~m}^{2}$ for a dynamic fluid viscosity of $10^{-3} \mathrm{~Pa} \cdot \mathrm{s}$ and a fluid unit weight of approximately $10 \mathrm{kPa} / \mathrm{m}$ (freshwater).

The techniques for estimating the permeability characteristics of a geologic material certainly depend on the expected range of permeabilities of the material and is, to an extent, influenced by the availability of suitable experimental facilities that can measure very low volume flow rates through the sample and the measurement of rapid fluid pressure changes at pressurized regions in contact with the sample. The most straightforward approach is to conduct steady state flow tests through a saturated sample; in this approach, the steady flow is governed by Laplace's equation (Bear, 1972; Selvadurai, 2000a) and regardless of the complexity of the experimental configuration, either analytical or computational solutions can be obtained to describe the flow process. Even complexities such as hydraulic orthotropy and hydraulic heterogeneity can be accommodated in the modeling of flow problems relevant to laboratory and field conditions (Selvadurai, 2004a, 2010; Selvadurai and Selvadurai, 2010, 2014). The parameters required for the interpretation of most experimental configurations are a set of stationary Dirichlet and/or Neumann boundary conditions on specified surfaces of the flow domain. The flow rate in the medium resulting from the prescribed potentials can then be related to permeability of the porous medium with the assumption of the usual relationship between the hydraulic conductivity and permeability. For low permeability materials, the time taken to initiate steady flow through the system can be excessive and the basic assumption of a completely saturated pore space may not be realized within the flow domain, which can influence the permeability estimation. Furthermore, the long duration of such experiments can lead to the release of dissolved air that can occlude pathways, which can lead to erroneous interpretations of the permeability. An approach that has been adopted is to minimize the flow paths or reduce the sample thickness with the intention of attaining steady flow relatively quickly. This procedure needs to be viewed with caution since relative pressure gradients across thin samples can result in stress states that may cause damage to the sample, including the opening of defects, which can influence the estimated permeability. The steady state permeability test, if it can be attained successfully, still represents the most effective method for estimating the permeability of low permeability materials since no other constitutive parameters related to the mechanical behaviour of the rock are involved in the estimation of permeability. The work of Bernaix (1969) is of particular interest since it was initiated after the failure of the Malpasset Dam in 1959 (France), and represents a contribution of significant merit in the use of steady state tests for measuring the permeability of rocks.

The time limitations associated with conducting steady state permeability tests on low permeability materials have prompted the use of transient tests where the permeability is estimated from a process involving time-dependent decay of an applied pressure field. The procedure is very similar to the estimation of the permeability of a fluid-saturated soil through observations of its consolidation behaviour (Terzaghi, 1923). The application of transient tests to estimate the permeability of rocks stems from the pioneering work done by Brace et al. (1968) who used the technique to determine the permeability reduction of Westerly Granite subjected to high confining pressures. Other studies of permeability alteration due to stress states are given by Zoback and Byerlee (1975), David and Darot (1989), David et al. (1994), Shiping et al. (1994), Kiyama et al. (1996), and Selvadurai and Głowacki (2008). Applications of damage mechanics-based interpretations of permeability evolution are given by Mahyari and Selvadurai (1998), Selvadurai and Shirazi (2004) and an extensive review of the influence of damage on poroelastic behaviour is given by Selvadurai (2004b). The basic philosophy in transient testing is that a fluid volume in contact with the saturated porous medium can be pressurized and the pressure will decay as the fluid migrates from the cavity to the porous domain. In principle, this is a sound test; however, there are a number of factors that can influence the pressure pulse decay. First, the region being tested is assumed to be fully saturated. If the porous medium has a low permeability, it will require time to achieve full saturation. Vacuum saturation of the porous medium is one approach that has been employed to reduce the saturation time; the disadvantage of this procedure is that residual pressure fields may be present in the sample, which can influence the pulse decay response and hence permeability estimation (Selvadurai, 2009). Furthermore, the time for dissipation of residual pressure fields will depend on the permeability of the porous medium, which is the parameter that needs to be determined. The process of pressure decay involves poroelastic responses (Biot, 1941; Selvadurai, 1996c, 2007; Verruijt, 2014) and the complete poroelasticity characteristics of the porous medium need to be determined in order to accurately interpret the results of pulse tests. These include an extensive array of deformability characteristics for both the porous skeleton and the saturating porous fluid. The reduced version of the poroelasticity equations 
were developed by Brace et al. (1968), resulting in a piezoconduction equation for the pore fluid pressure. The piezo-conduction equation is derived by imposing certain restrictions on the mechanical response of the skeletal deformations without excluding the compressibility effects indicated previously. The parameters controlling the pressure decay include the compressibility of the porous skeleton, the compressibility of the grains, the compressibility of the pore fluid and the porosity of the porous skeleton. The influences of these restrictions, in comparison with the complete Biot result for the pore pressure decay response, were compared by Hart and Wang (1998) who examined the onedimensional piezo-conduction equation. More recently Selvadurai and Najari (2013) presented a comparison of the two approaches by examining the responses of rocks such as Indiana Limestone and Westerly Granite and supplemented the study with experimental results obtained from Stanstead Granite. The parameters controlling the pressure decay can, in general, be determined relatively accurately from conventional geotechnical tests. The estimation of porosity for low permeability materials is non-routine; the conventional water saturation techniques are time consuming and measurements using mercury intrusion porosimetry provide more reliable estimates. Other major factors that can influence the piezo-conduction equation response are the compressibility of the pore fluid which resides in the pore space of the rock and the fluid in the cavity that is being pressurized. This aspect is rarely discussed in research related to the permeability measurement of low permeability materials. Selvadurai and Ichikawa (2013) examined the role of partial saturation of the pore fluid on the one-dimensional cavity pressure decay response and showed that the role of unsaturation becomes more important when the air fraction is large and it is particularly important when dissolved air and air solubility effects are taken into consideration (Selvadurai, 2009). The study by Selvadurai and Najari (2015) examined the role of an air fraction in the pressurized cavity on the pulse decay response; the volume of the air fraction can be estimated by recording the cavity pressurization response and the pulse decay response can be corrected to account for the effective compressibility of the pressurized fluid. These studies show that when the hydraulic pulse test results are corrected for the air fraction, they correspond closely to the results from steady state tests, which can provide the most accurate permeability estimates. Of related interest are recent studies by Jannot and Lasseux (2012) and Golfier et al. (2015) that focus on the use of gas permeability tests for the estimation of permeability of respectively, weakly permeable media and vuggy sandstone, taking into consideration alternative laws for the pore fluid migration.

This paper discusses the experimental procedures that have been developed for conducting both steady state and transient hydraulic pulse tests on cylindrical samples of the
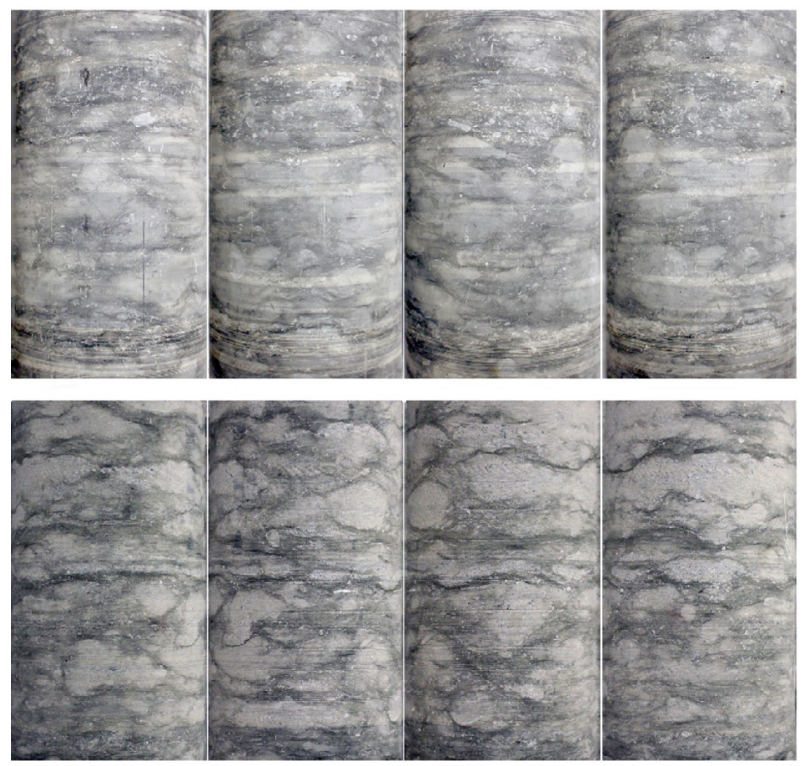

Figure 2

The Cobourg Limestone samples cored normal to the plane of nominal stratification (CL-H1: upper, CL-H2: lower).

argillaceous Cobourg Limestone and presents results that indicate that cavity air fraction and axial stresses can influence the estimation of the permeability of the rock.

\section{EXPERIMENTAL APPROACHES}

This paper presents the results of permeability tests conducted on 4 cylindrical samples of the Cobourg Limestone measuring $150 \mathrm{~mm}$ in diameter and $278 \mathrm{~mm}$ in length. Two of the test specimens were cored normal to the nominal clay partings, i.e. CL-H1, CL-H2 (Fig. 2) and the other two parallel to the nominal clay partings, i.e. CL-V1, CL-V2 (Fig. 3). Each sample contains a concentrically located cylindrical cavity of diameter $26 \mathrm{~mm}$ and length $139 \mathrm{~mm}$, which serves as the pressurized fluid cavity for conducting the hydraulic pulse tests. The cavity was formed using a diamond tipped corer, which provided a smooth surface to minimize air accumulation during filling. The test arrangement is not dissimilar to that described by Bernaix (1969) for performing radial flow steady state tests on Miliolite Limestone and Malpasset Gneiss. However, computational approaches were used in this study to examine steady state tests and a novel application of computational approaches was used to examine the developments in transient hydraulic pulse testing.

The primary requirement of any experimental technique developed for estimating the permeability of porous geomaterials is that the test arrangement should be free of any 

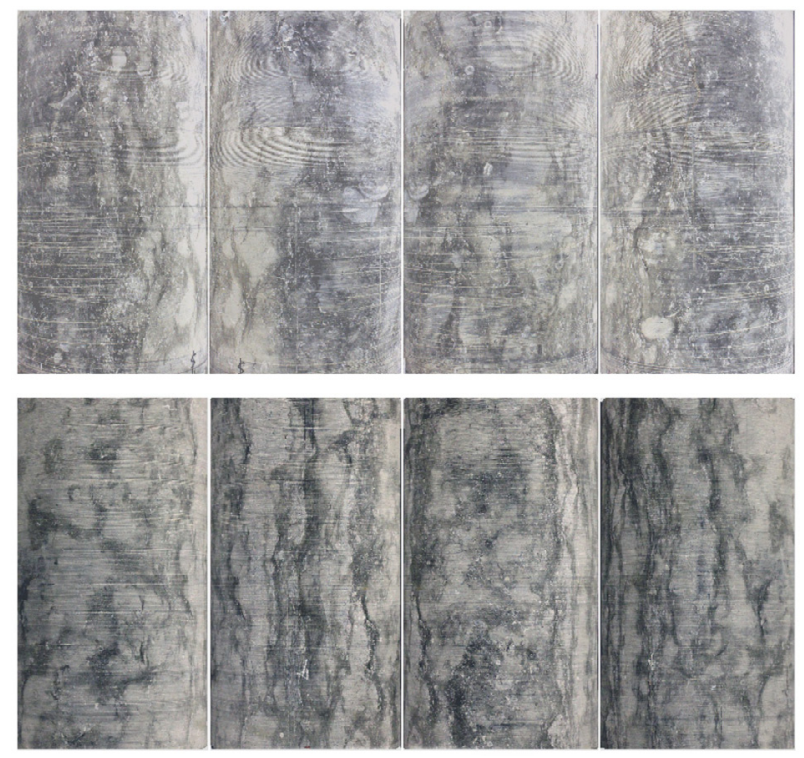

Figure 3

The Cobourg Limestone samples cored parallel to the plane of nominal stratification (CL-V1: upper, CL-V2: lower).

leakages from interfaces and fittings that could contribute to an erroneous interpretation of the permeability. This experimental requirement was investigated extensively in connection with the testing of the Cobourg Limestone and Stanstead Granite (Selvadurai et al., 2011; Selvadurai and Jenner, 2012; Selvadurai and Najari, 2013, 2015). If the hydraulic pulse permeability tests are conducted in an environment where the sample can be subjected to triaxial stress states, leakage from the pressurized cavity, through the membrane which isolates the sample, can be eliminated by the application of suitable confining stresses. When permeability testing is carried out in an unstressed condition, it is necessary to adopt an alternative sealing technique. Trial experiments indicated that the use of epoxy sealing techniques provides the most impervious sealing and the reliability of the procedures have been verified by conducting tests using pressurizing devices that were epoxy glued to stainless steel surfaces. Several epoxy glues were tested and the marine epoxy supplied by LePage ${ }^{\mathrm{TM}}$ provided the most reliable seal. The epoxy provided a stable seal over the temperature range of $-23^{\circ} \mathrm{C}$ to $150^{\circ} \mathrm{C}$ and the maximum pressure required to initiate interface delamination was $13 \mathrm{MPa}$ (Jenner, 2012). The partially drilled cavity implied that only the entrance to the cavity required sealing; a stainless steel plate with a thickness of $0.64 \mathrm{~cm}$ with fittings was epoxy glued to the plane end of the cylindrical sample (Fig. 4). The same epoxy was used to seal all the fittings and fixtures used in the experimental arrangements. The fixtures included water entry and exit ports, two thermocouples that measured the temperature of the water in the top and the bottom of the cavity and an in-line pressure transducer (a $1 \mathrm{MPa}$ Omega pressure transducer; Model: MMG150V5K4C1T4A6S) that was capable of measuring the cavity pressure to an accuracy of $0.2 \%$ of the full range. The placement of the thermocouples followed the procedures developed in previous research investigations by Selvadurai (1996a, 2002); the wires that passed through the fittings were sealed with marine epoxy to eliminate moisture loss and leakage. A schematic view of the experimental setup is shown in Figure 5.

Any change in the ambient temperature can affect the estimation of permeability of a low permeability rock in two main ways:

- the viscosity of the water which controls the flow rate through the pore space is sensitive to temperature changes;

- any changes in temperature can induce excess pore pressure in the rock due to the differential thermal expansion of the pore water and the porous skeleton and affect the flow rate through the rock.

In order to minimize the influence of temperature change on the results of the permeability tests, the temperature of the water in the container where the sample was placed was maintained constant at $25^{\circ} \mathrm{C} \pm 0.05^{\circ} \mathrm{C}$. The experimental setup was capable of maintaining a constant temperature by refilling the container from a water reservoir regulated by a solenoid valve connected to a temperature controller.

\section{EXPERIMENTAL PROCEDURES}

Prior to performing either steady state or transient hydraulic pulse tests, each Cobourg Limestone sample with cavity sealing was subjected to vacuum saturation for a period of 7 days at a vacuum of $-85 \mathrm{kPa}$. This ensured that regions of the rock close to the surfaces being pressurized during permeability testing were in a near-saturated condition. The samples were kept in water under zero vacuum to ensure that any residual pressures dissipated prior to conducting either steady state or transient hydraulic pulse tests. Each sample was then assembled in a test frame capable of applying axial stresses to the sample ranging from 1.0 MPa to 2.5 MPa. The controlled axial loads were applied using a nitrogen pressurized accumulator; the axial load was applied in order to minimize the possibility of delamination of the stainless steel plate that was epoxied to the bottom of the sample and to investigate the alteration of the permeability characteristics of the rock to confinement.

The fluid flow necessary for performing either steady state tests or hydraulic pulse tests was provided by a precision Quizix Pump (QX-6000) capable of supplying flow rates 
a)

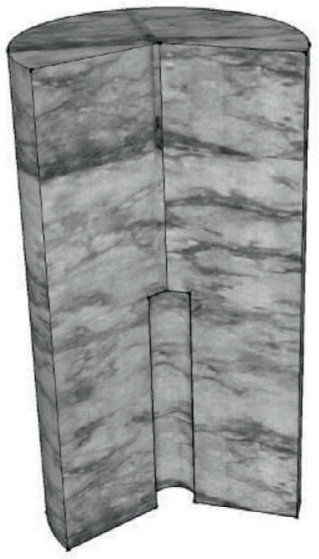

c)

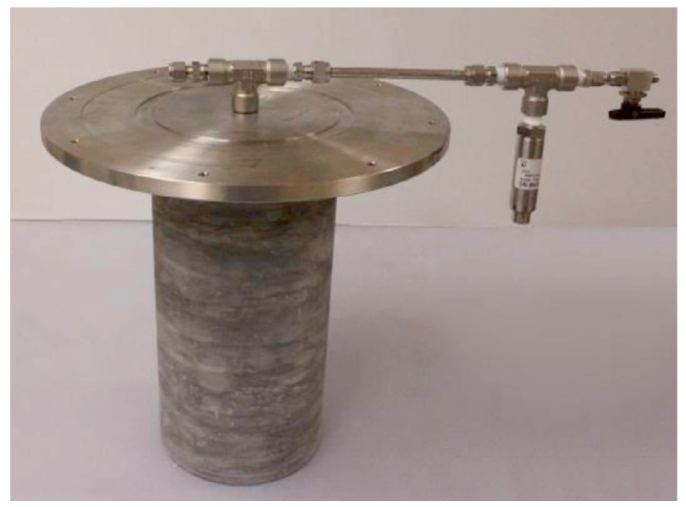

b)

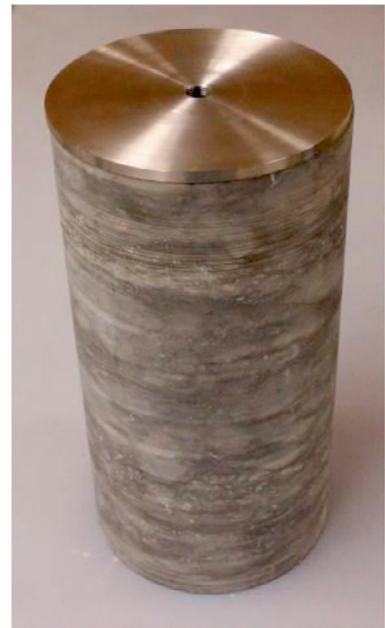

d)

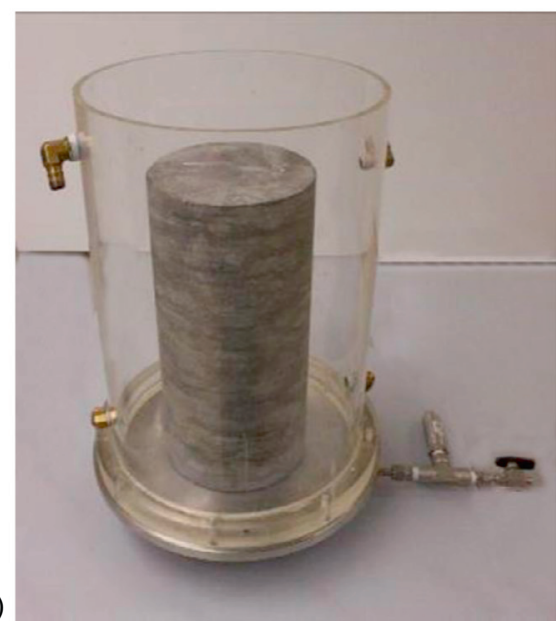

Figure 4

The central cavity in the Cobourg Limestone and the experimental arrangements: a) sample CL-H1 showing partially drilled central cavity, b) the epoxied stainless steel plate, c) the base plate of the reservoir containing the fixtures, d) the assembled sample in a container.

ranging from $0.0003 \mathrm{~mL} / \mathrm{min}$ to $50 \mathrm{~mL} / \mathrm{min}$. The pump uses a dual piston system in order to guarantee a continuous flow. Smaller flow rates can be measured by applying a constant pressure to the sample and measuring the flow rate required to maintain the pressure. The pump is capable of measuring any change in the volume of each piston down to $1 \mathrm{~nL}$. The temperatures within the pressurized cavity and the pressure within the cavity were recorded for each test using an Instrunet $^{\mathrm{TM}}$ data acquisition integrated with a computer.

\subsection{Steady State Tests}

The permeability of the sample was estimated using a constant pressure steady state technique. A constant pressure of $100 \mathrm{kPa}$ was applied to the fluid-filled cavity using a
Quizix pump and the flow rate required to maintain the water pressure was monitored. Although the water pressure in the cavity is well below the critical pressure for delamination of the epoxy (13 MPa), a $1 \mathrm{MPa}$ axial sealing pressure was applied to the sample in order to minimize any possible delamination. In order to examine any leakage through the fittings, the entry valve to the central cavity was closed and the sealing performance of the fittings between the pump pistons and the valve was investigated. The test was continued for 2 days and the average leakage through the fittings was estimated to be $0.5 \times 10^{-6} \mathrm{~mL} / \mathrm{min}$ at $100 \mathrm{kPa}$ constant pressure. Constant pressure steady state tests were performed on each of the four Cobourg Limestone samples and the results used to estimate the permeability. The results of the tests are shown in Figures 6 and 7. 


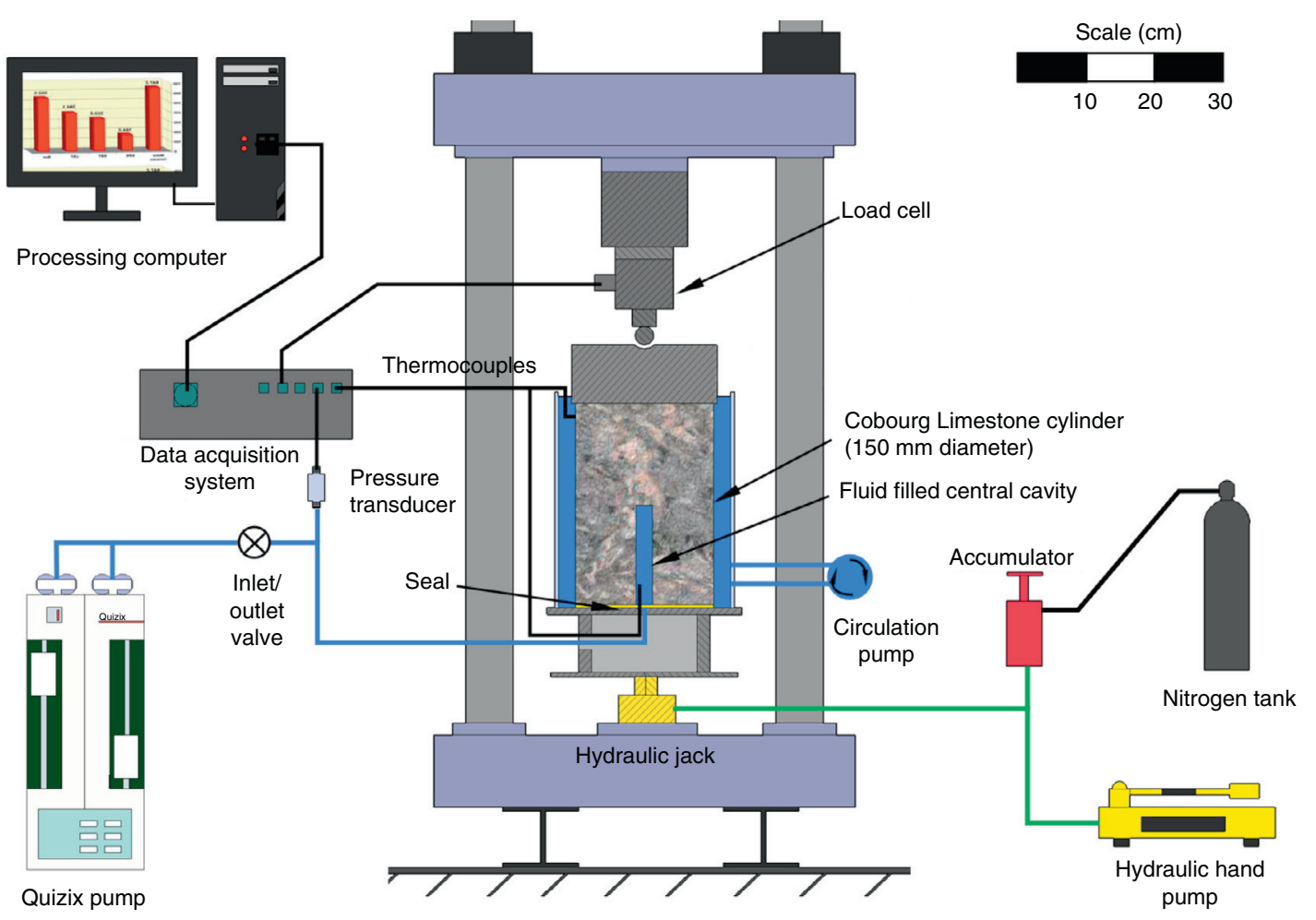

Figure 5

A schematic view of the experimental facility.

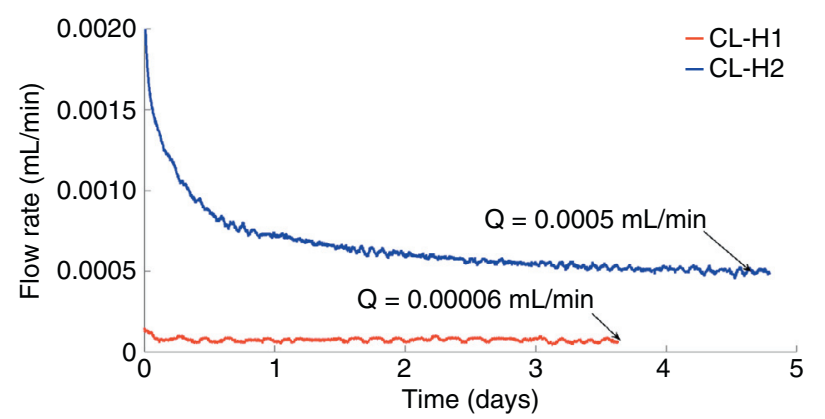

Figure 6

The results of permeability tests performed on cylindrical samples cored normal to the bedding plane, CL-H1, CL-H2.

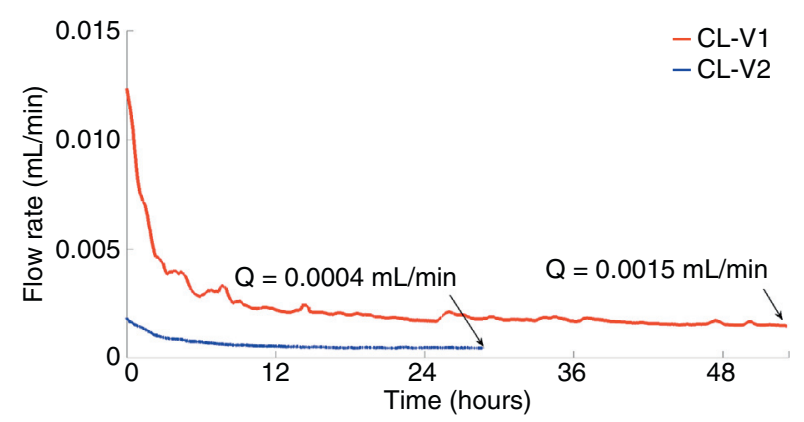

Figure 7

The results of permeability tests performed on cylindrical samples cored parallel to the bedding plane, CL-V1, CL-V2.

\subsection{Analysis of the Test Results}

The mass conservation equation for a rigid porous medium saturated with an incompressible fluid is (Selvadurai, 2000a):

$$
\nabla \cdot \mathbf{v}=0
$$

where $\mathbf{v}$ is the fluid velocity vector. For flow through an isotropic, homogeneous, non-deformable porous medium that can be described by Darcy's law, the flow velocity is given by:

$$
\mathbf{v}=-\frac{K}{\mu} \nabla\left(p+\gamma_{w} D\right)
$$


where $\gamma_{w}$ is the specific unit weight of water, $\mu$ is the dynamic viscosity of water, $K$ is the isotropic permeability, $\nabla$ is the gradient operator, $D$ is the datum head and $p$ is the pore fluid pressure. Substituting Equation (2) in Equation (1), we obtain Laplace's equation for steady flow through the porous medium as:

$$
\nabla^{2} p=0
$$

Laplace's equation for an axisymmetric flow referred to a cylindrical polar coordinate system takes the form:

$$
\frac{\partial^{2} p}{\partial r^{2}}+\frac{1}{r} \frac{\partial p}{\partial r}+\frac{\partial^{2} p}{\partial z^{2}}=0
$$

The steady state flow permeability tests performed on the Cobourg Limestone cylinders can be modeled using Equation (4). The boundary conditions applicable for potential flow in a cylindrical sample of radius $b$ and height $H$ containing a co-axial cylindrical cavity of radius $a$ and length $l$ (Fig. 8) can be written as:

$$
\begin{aligned}
& \frac{\partial p}{\partial r}=0 ; \quad r=0 ; 0 \leq z \leq H \\
& \frac{\partial p}{\partial z}=0 ; \quad a<r<b ; z=0 \\
& p=p_{0} ; \quad r=a ; 0 \leq z \leq l \\
& p=p_{0} ; \quad 0 \leq r \leq a ; z=l \\
& p=0 ; \quad 0 \leq r \leq b ; z=H \\
& p=0 ; \quad r=b ; 0 \leq z \leq H
\end{aligned}
$$

where the base surface of the cylinder is sealed, a constant pressure $p_{0}$ is applied to the surface of the central cavity and the outer surface of the cylinder is kept at atmospheric pressure.

A complete analytical solution to the above boundary value problem can be developed using the theory of integral transforms applicable to the axisymmetric cylindrical polar coordinate formulation of problems in potential theory (Sneddon, 1951; Tranter, 1971; Selvadurai, 2000b). The solution of the resulting integral equations is non-routine (Selvadurai and Singh, 1984, 1985) and also involves computational approaches. Instead, we develop an approximate analytical result by imposing Neumann boundary conditions on the continuous material plane at the end of the cylindrical cavity; i.e.:

$$
\frac{\partial p}{\partial z}=0 ; a \leq r \leq b ; z=l
$$

which divides the flow into two regions: (i) a purely radial flow region in the domain $r \in(a, b), z \in(0, l)$ and (ii) axisymmetric flow from a circular patch into a halfspace region $r \in(0, \infty) ; z \in(l, \infty)$ (with the exterior region completely

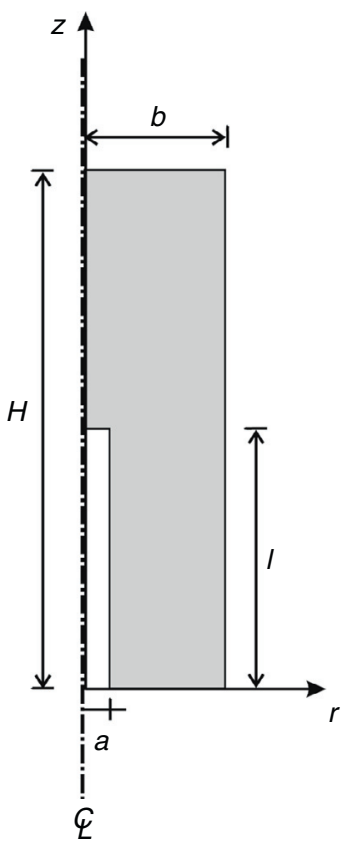

Figure 8

The geometry of the Cobourg Limestone specimens tested for permeability measurement.

sealed with the boundary condition in Equation (6), and the interior region subjected to a constant potential $p_{0}$ ). For the sub-problem (i), the steady rate at which the water flows through a hollow cylinder of length $l$, where the inner cylindrical surface at $r=a$ is subjected to a constant pressure of $p_{0}$ and the outer cylindrical surface is kept at atmospheric pressure, can be evaluated in the form (Selvadurai, 2000a):

$$
Q_{R}=\frac{2 \pi l K p_{0}}{\mu \log _{e}\left(\frac{b}{a}\right)}
$$

An analytical study of the fluid flow into a half-space through a pressurized circular aperture at a sealed surface is given by Selvadurai (2010) and Selvadurai and Selvadurai (2010). The study by Selvadurai and Selvadurai (2010) developed a "Patch Permeability Test", which involves an annular sealed region, to estimate the effective permeability of a cuboidal sample. The mathematical solution was developed using the results given by Selvadurai and Singh (1984, 1985). In the particular instance when the sealed region of the surface of the halfspace region corresponds to $r \in(a, \infty)$, the flow rate through the circular patch can be evaluated in the exact closed form:

$$
Q_{P}=\frac{4 a K p_{0}}{\mu}
$$


TABLE 1

Permeability of Cobourg Limestone samples estimated from constant pressure steady state test results

\begin{tabular}{c|c|c|c}
\hline Sample & $Q(\mathrm{~mL} / \mathrm{min})$ & $K\left(\mathrm{~m}^{2}\right)^{*}$ & $K\left(\mathrm{~m}^{2}\right)^{* *}$ \\
\hline CL-H1 & 0.00006 & $1.62 \times 10^{-20}$ & $1.56 \times 10^{-20}$ \\
\hline CL-H2 & 0.00050 & $1.35 \times 10^{-19}$ & $1.30 \times 10^{-19}$ \\
\hline CL-V1 & 0.00150 & $4.05 \times 10^{-19}$ & $3.9 \times 10^{-19}$ \\
\hline CL-V2 & 0.00040 & $1.08 \times 10^{-19}$ & $1.04 \times 10^{-19}$ \\
\hline
\end{tabular}

* Permeability value estimated from the approximate analytical result [9].

** Permeability value estimated from computational modeling using COMSOL Multiphysics ${ }^{\mathrm{TM}}$.

The approximate analytical result for the flow rate through the pressurized cavity can be evaluated by combining Equations (7) and (8); i.e.:

$$
Q=\left(4+\frac{2 \pi\left(\frac{l}{a}\right)}{\log _{e}\left(\frac{b}{a}\right)}\right) \frac{a K p_{0}}{\mu}
$$

The permeability of the Cobourg Limestone samples can be estimated using Equation (9). In order to examine the accuracy of the approximate analytical solution to the potential flow problem for a sample with a partial cylindrical cavity, the problem was also solved using the finite element software COMSOL Multiphysics ${ }^{\mathrm{TM}}$ for specified geometry and boundary conditions. The model contained 20908 quadratic triangular elements with Lagrange shape function and 42247 degrees of freedom. In the current experimental investigations, the dimensions of the samples are as follows: $H=278 \mathrm{~mm}, l=139 \mathrm{~mm}, a=13 \mathrm{~mm}, b=75 \mathrm{~mm}$. Given the water pressure and flow rate at the intake under steady state condition for each sample, the permeability of the samples was estimated using both the appropriate analytical and computational techniques (Tab. 1). Figure 9 shows the finite element mesh used in the modeling along with the flow net and the pore pressure distribution in the flow domain.

\subsection{Steady State Tests-Axial Stress Cycle}

Cobourg Limestone specimens used in the present study showed some evidence of discontinuities at locations in the vicinity of the clay partings. In order to examine the influence of these discontinuities on the permeability characteristics of the rock, the permeability of the Cobourg Limestone samples CL-H2 and CL-V2 were examined under various uniaxial compressive loads. An axial load was applied along the central axis of the cylinders. The permeability of each sample was examined for three different stress conditions: a) $1 \mathrm{MPa}$ axial stress, b) $2.5 \mathrm{MPa}$ axial stress and c) $1 \mathrm{MPa}$ axial stress after unloading from stage $b$ ).

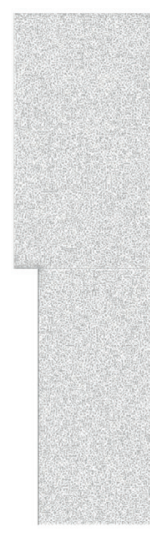

a)

Figure 9

Computational simulation of the test arrangement. a) The mesh used in the finite element model, b) the flow net, c) the contours of the pore fluid pressure.

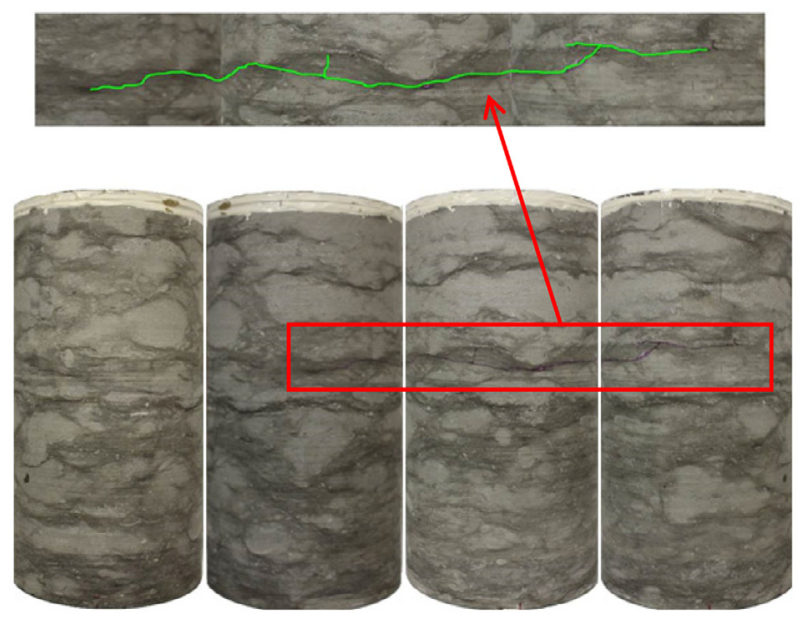

Figure 10

The seepage of dye from the central fluid-filled cavity through a discontinuity in sample CL-H2.

Prior to performing the permeability tests under different stress conditions, the central cavity of the samples was filled with a water-soluble dye (potassium permanganate). A constant pressure of $100 \mathrm{kPa}$ was then applied to the central cavity for 24 hours, with the sample maintained in an axially unstressed condition. Seepage of the dye through a discontinuity was observed in sample CL-H2 (Fig. 10) but there was no evidence of dye from other sections of the surface of the sample CL-V2. After performing the dye test, the dye in the central cavity was replaced with water and the samples were re-saturated in a vacuum chamber before performing the 


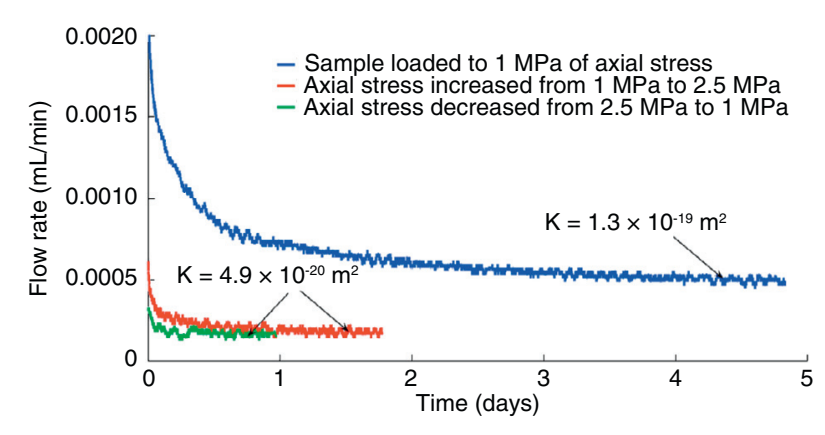

Figure 11

The results of permeability tests performed on the Cobourg Limestone cylinder CL-H2 under different compressive stress values.

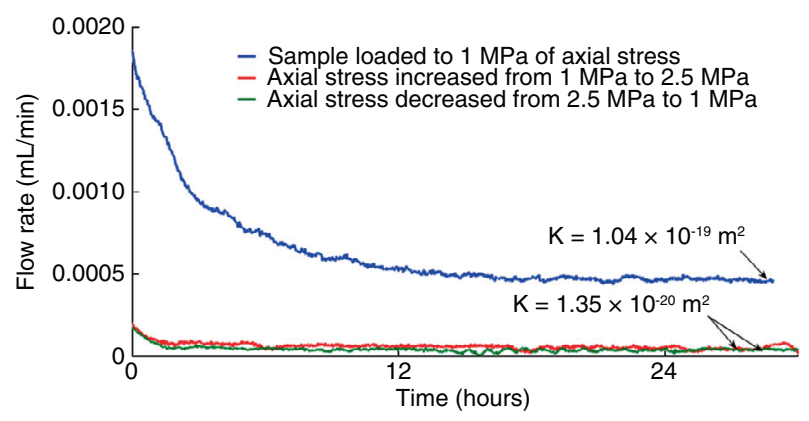

Figure 12

The results of permeability tests performed on the Cobourg Limestone cylinder CL-V2 under different compressive stress values.

permeability tests under different axial compressive stresses. The samples were placed inside a water chamber in the loading frame and an axial stress was applied using a nitrogen pressurized accumulator. The results of the experiments along with the estimated permeability values under each loading stage for the samples CL-H2 and CL-V2 are shown in Figures 11 and 12, respectively. The results show that an increase in the axial stress from $1 \mathrm{MPa}$ to $2.5 \mathrm{MPa}$ decreased the permeability from $1.3 \times 10^{-19} \mathrm{~m}^{2}$ to $4.9 \times 10^{-20} \mathrm{~m}^{2}$ in sample CL-H2 and from $1.04 \times 10^{-19} \mathrm{~m}^{2}$ to $1.35 \times 10^{-20} \mathrm{~m}^{2}$ in sample CL-V2. It was also observed that the measured permeability did not return to its original value upon reduction of the axial stress from 2.5 MPa to $1 \mathrm{MPa}$. The permanent permeability reduction due to the application of the axial stress cycle can be attributed to any one of the following factors:

- irreversible closure of fissures or microcracks,

- crushing of the asperities composing the defect,

- swelling of the argillaceous component of the rock, suggesting possible evidence of a healing mechanism.

The results of the research are preliminary and, as such, cannot provide any definite conclusions on stress-assisted permeability reduction in the Cobourg Limestone, but they merit further investigation.

\section{HYDRAULIC PULSE TESTS}

The permeability of Cobourg Limestone was also examined by performing a series of hydraulic pulse tests on two of the samples tested above, cylindrical sample CL-H1 with horizontally oriented argillaceous partings and the cylindrical sample CL-V2 with vertically oriented argillaceous partings. The pulse tests on sample CL-H1 were performed after conducting a steady state constant pressure test and the pulse tests on CL-V2 were performed after the sample underwent an axial loading-unloading cycle (Sect. 2).

The hydraulic pulse tests involved the application of a pressure pulse to the fluid-filled central cavity of each Cobourg Limestone sample by pumping water at a constant rate. The inlet valve was then closed and the fluid cavity pressure dissipation was monitored. In order to minimize the effect of ambient temperature changes on the pressure decay curves, the temperature was kept at $25 \pm 0.05^{\circ} \mathrm{C}$. The cavity pressure was first increased to $100 \mathrm{kPa}$ and kept constant for 3 days until it reached a steady state. The cavity pressure was then increased from $100 \mathrm{kPa}$ to $300 \mathrm{kPa}$ by applying a constant flow rate of $0.1 \mathrm{~mL} / \mathrm{min}$. The inlet valve was then closed and the cavity pressure was allowed to gradually dissipate back to $100 \mathrm{kPa}$. At this point, the cavity pressure was brought back to $300 \mathrm{kPa}$ by pumping water into the cavity at the same flow rate. The same procedure was repeated for 5 hydraulic pulse tests on each of the two samples. Since the compressibility of the pressurized fluid is low, a minimal migration of fluid to the porous medium results in a pressure drop in the cavity. The compressibility of de-aired water at $20^{\circ} \mathrm{C}$ is $4.54 \times 10^{-10} \mathrm{~Pa}^{-1}$ (White, 1986), which is defined as the relative volume decrease induced by the pressure increase. Although the central cavity and the attached connections were filled with water and precautions were taken to minimize the presence of air in the cavity, it is almost impossible to completely eliminate the air fraction in the pressurization volume. A practical technique was proposed by Selvadurai and Najari (2015), which takes into account the effect of air fraction on the analysis of the hydraulic pulse test results. The technique uses the pressure build-up curves to estimate the volume of air fraction and then adjusts the compressibility of the cavity fluid accordingly. Neglecting the influences of solubility of air in water, the surface tension of water and the vapour pressure, the compressibility of the air-water mixture can be written as:

$$
C_{e q}=\frac{\varphi}{P}+(1-\varphi) C_{w}
$$




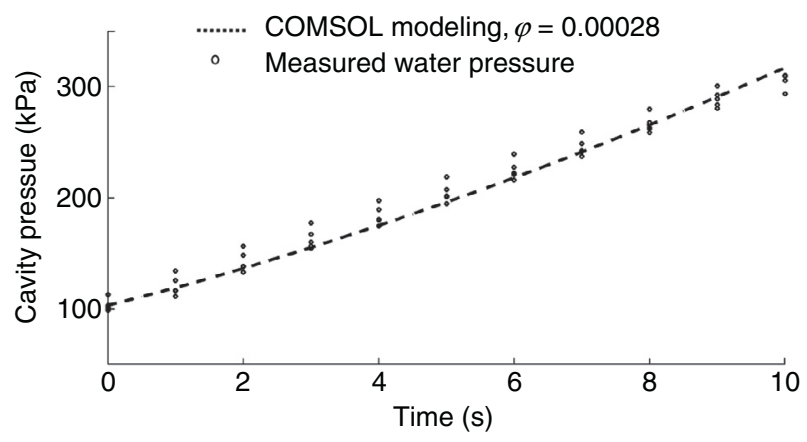

Figure 13

The pressure build-up curves of the hydraulic pulse tests performed on sample CL-H1.

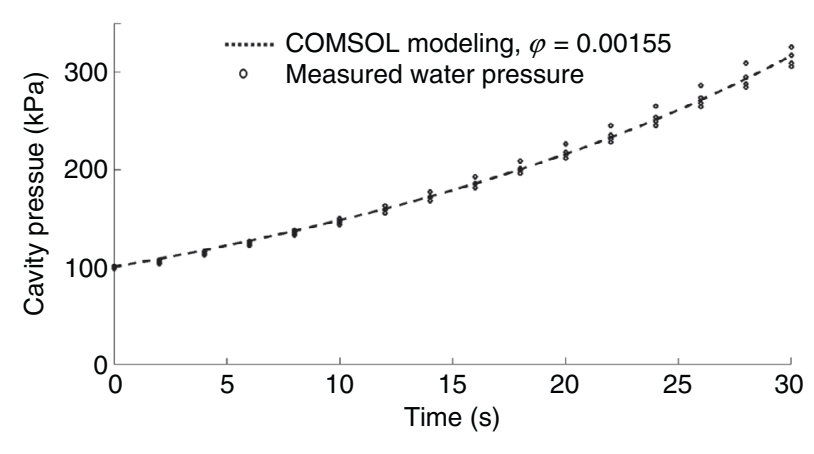

Figure 14

The pressure build-up curves of the hydraulic pulse tests performed on sample CL-V2.

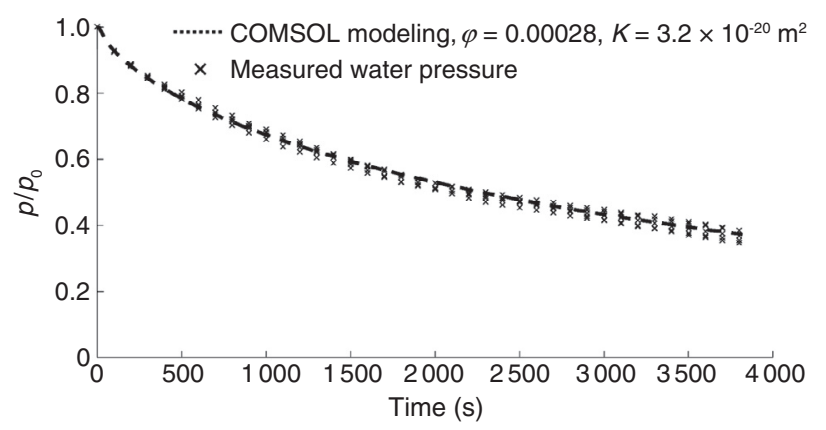

Figure 15

The pressure decay curves of the hydraulic pulse tests performed on sample CL-H1.

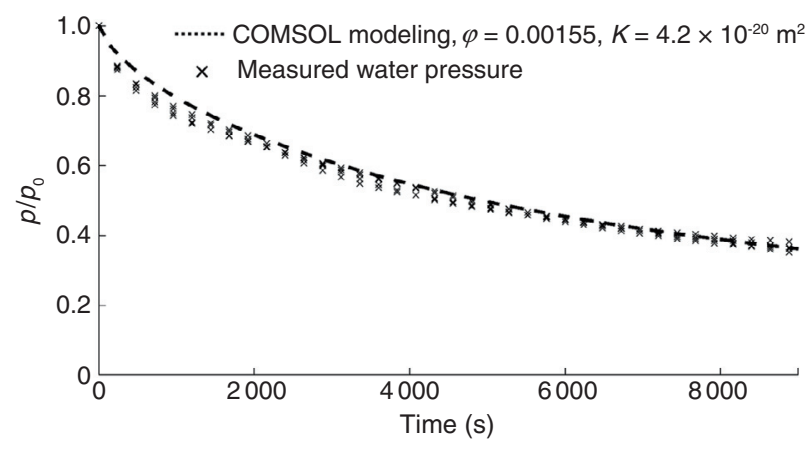

Figure 16

The pressure decay curves of the hydraulic pulse tests performed on sample CL-V2. where $C_{e q}$ is the compressibility of the air-water mixture, $\varphi$ is the air fraction, $P$ is the absolute water pressure and $C_{w}$ is the compressibility of de-aired water. The compressibility of the air-water mixture can then be incorporated in the piezoconduction equation:

$$
\left\{n C_{e q}+C_{e f f}-(n+1) C_{s}\right\} \frac{\partial p}{\partial t}-\frac{K}{\mu} \nabla^{2} p=0
$$

where $C_{e f f}$ is the compressibility of the porous skeleton $C_{s}$ is the compressibility of the solid grains, $n$ is the porosity, $K$ is the permeability and $\mu$ is the dynamic viscosity of the water. The compressibility of the porous skeleton and the solid grains can be written in terms of three poro-elasticity parameters:

$$
C_{e f f}=\frac{3(1-2 v)}{E} ; C_{s}=(1-\alpha) C_{e f f}
$$

where $E$ is the Young's modulus, $v$ is the Poisson's ratio and $\alpha$ is the Biot coefficient.
The pressure build-up curves for the hydraulic pulse tests performed on samples CL-H1 and CL-V2 are shown in Figures 13 and 14 respectively. The pressure build-up stage was modeled in COMSOL in order to estimate the air fraction for each sample. The parameters used in the modeling were as follows (Selvadurai et al., 2011; Wang, 2000): the skeletal Young's modulus $E=21 \mathrm{GPa}$, the Poisson's ratio $v=0.25$, Biot's coefficient $\alpha=0.7$ and porosity $n=1 \%$ to $4 \%$. It was observed that the curves with an air fraction of 0.00028 , and 0.00155 provided the best fit with the recorded pressure build-up curves for samples CL-H1 and CL-V2, respectively. The pressure decay curves were then analyzed using the modified pressure-dependent compressibility equation for the fluid in the pressurized cavity (Fig. 15, 16). Using the modified computational scheme, the permeability values were estimated to be $K=3.2 \times 10^{-20} \mathrm{~m}^{2}$ and $4.2 \times 10^{-20} \mathrm{~m}^{2}$ for samples CL-H1 and CL-V2, respectively; these results compare favorably with the results obtained from steady state test results. 


\section{DISCUSSION}

There are several factors that could introduce errors in the estimation of permeability. These factors can be categorized into two groups:

- errors associated with the measurement of flow rate and fluid pressure,

- alteration of the hydraulic characteristics of the rock due to mechanical or chemical phenomena while permeability tests are being conducted.

The main parameters that could influence the measurement of the flow rate and fluid pressure are the leakage through the fittings, the sealing condition and the accuracy of the electronic devices measuring the parameters. The efficiency of using epoxy in sealing Cobourg Limestonestainless steel interface was extensively studied by Jenner (2012). The leakage through the fittings, estimated by performing a constant pressure steady state test was approximately $0.5 \times 10^{-6} \mathrm{~mL} / \mathrm{min}$. Since the minimum flow rate measured throughout the tests performed was $4 \times 10^{-5} \mathrm{~mL} / \mathrm{min}$, the influence of the leakage on the estimation of permeability was considered to be negligible. Regarding the accuracy of the electronic devices used in the experiments, the pressure transducer measured the pressure to within an accuracy of $\pm 0.2 \%$ of the full range, i.e. $\pm 2 \mathrm{kPa}$ and the pump measured any change in the volume of each piston to an accuracy of approximately $1 \mathrm{~nL}$. These processes introduced negligible errors in the estimation of the permeability.

The alteration of the hydraulic properties of the rock due to mechanical or chemical phenomena while permeability tests are being conducted can also influence the estimation of the permeability parameter. Examples of such phenomena and their possible influences, e.g. damage induced during application of water pressure during a test, swelling of the constituent components of rocks, self-healing mechanisms of fractures and microcracks, were briefly discussed in the manuscript. A detailed study of such phenomena was not within the scope of the current research and each aspect requires further investigation. The repeatability of the pressure decay results obtained from the hydraulic pulse tests performed on samples CL-H1 and CL-V2, however, verified negligible influences of possible mechanical or chemical phenomena in the current experimental setup with the fluid pressures ranging from 0 to $300 \mathrm{kPa}$.

Apart from the above explanations, the results of the constant pressure steady state tests performed on the four different samples estimated the permeability to be within $1.56 \times 10^{-20} \mathrm{~m}^{2}$ to $1.04 \times 10^{-19} \mathrm{~m}^{2}$ which is a narrow range, given that the inhomogeneities and stratifications of the rock that are visible. The permeability estimated from the hydraulic pulse tests performed on two of the samples were within the range $3.2 \times 10^{-20} \mathrm{~m}^{2}$ to $4.2 \times 10^{-20} \mathrm{~m}^{2}$ which also is within the same order of magnitude of the results obtained from the steady state tests.

\section{CONCLUDING REMARKS}

The permeability characteristics of the heterogeneous argillaceous Cobourg Limestone were examined by performing a series of constant pressure steady state tests on 4 cylindrical samples. The fabric of the Cobourg Limestone is heterogeneous with inter-bedding of an argillaceous component contributing to a nominally stratified heterogeneity. There is reported evidence of nominal hydraulic transverse isotropy when testing one-dimensional specimens cored in the appropriate orientations (Vilks and Miller, 2007; Selvadurai et al., 2011; Selvadurai and Jenner, 2012). In the current experimental arrangements, the dominant flow direction is along the nominal argillaceous partings and the values of the permeabilities are indicative of the in-plane permeability. Also, at the scale of testing, the results of the tests performed are indicative of the average permeability of the Cobourg Limestone. The two samples tested were cored normal to the nominal bedding plane of the argillaceous partings while the other two samples were cored parallel to the bedding plane. The permeability of the samples cored normal to the plane of the argillaceous partings was estimated to be between $1.56 \times 10^{-20} \mathrm{~m}^{2}$ and $1.3 \times 10^{-19} \mathrm{~m}^{2}$ and the permeability of the samples cored along the argillaceous partings was estimated to be between $1.04 \times 10^{-19} \mathrm{~m}^{2}$ to $3.9 \times 10^{-19} \mathrm{~m}^{2}$. A simplified analytical estimate was developed to analyze the steady state results obtained from steady flow tests conducted with a pressurized cylindrical cavity occupying a part of the tested cylinder. The accuracy of the analytical modeling of the steady state permeability tests was evaluated by comparing the results with the analogous results obtained from the computational modeling of the problem using COMSOL Multiphysics ${ }^{\mathrm{TM}}$. It was seen that the simplified analytical equation can be used for the sample geometry in the experimental configuration with negligible error. The alteration of the permeability of the rock was also studied when two samples were subjected to uniaxial loading and unloading; these samples were cylinders cored normal to the nominal argillaceous partings and parallel to the partings. It was observed that the permeability of the samples experienced a decrease of between 2.6 to 7.7 times, when the uniaxial stress increased from 1.0 MPa to 2.5 MPa. Furthermore, there was no evidence of recovery of the permeability estimate during a subsequent loading-unloading cycle. The decrease in the permeability can most likely be attributed to several processes including closure of micro-cracks and discontinuities or the swelling of the argillaceous phase during water uptake. The permeability of the Cobourg Limestone was also estimated using a hydraulic pulse testing 
technique. The computational approach was used for the analysis of the test and the interpretation of the tests included the consideration of the effect of the air fraction within the pressurized cavity, which was estimated using the results of the cavity pressurization curve. The permeability values for the two samples tested ranged between $3.2 \times 10^{-20} \mathrm{~m}^{2}$ to $4.2 \times 10^{-20} \mathrm{~m}^{2}$ and compared favourably with the steady state test results performed on the same samples. Both the "Patch Permeability Test" (Selvadurai and Selvadurai, 2010) and the current "Partial Cavity Test" (Selvadurai and Najari, 2015) are innovative approaches for conducting steady state and transient pulse tests for estimating the fluid transport characteristics of low permeability geomaterials.

\section{ACKNOWLEDGMENTS}

The work described in this paper was initiated through a Discovery Grant awarded by the Natural Sciences and Engineering Research Council (NSERC) of Canada and through a Research Grant Awarded by the Nuclear Waste Management Organization, Ontario. The paper was prepared as a result of the Conference on The Challenge of Studying Low Permeability Materials, In Situ (field) and Numerical Methods, held in 2014 at The Universite Cergy Pontoise, Paris (France). The first author would like to express his thanks to Professor Christian David and Professor Jerome Wassermann for the invitation to present a Keynote Lecture at this meeting. The authors are indebted to Mr. Mark Jensen, Director, Deep Geologic Repository Geosciences and Research, Nuclear Waste Management Organization, Toronto, ON, Canada for his valuable comments. The comments of the reviewers, which led to improvements in the presentation, are also gratefully acknowledged.

\section{DISCLAIMER}

The use of the computational code COMSOL Multiphys$\mathrm{ics}^{\mathrm{TM}}$ is for demonstrational purposes only. The authors neither advocate nor recommend the use of the code without conducting suitable validation procedures to test the accuracy of the code in a rigorous fashion.

\section{REFERENCES}

Aldea C.-M., Shah S.P., Karr A. (1999) Permeability of cracked concrete, Materials and Structures/Matériaux et Constructions 32, 370-376.

Alonso E., Gens A. (Eds.) (2002) Key Issues in Waste Isolation Research, Engng. Geol. 64, 89-316.
Alonso E.E., Alcoverro J., Coste F., Malinsky L., MerrienSoukatchoff V., Kadiri I., Nowak T., Shao H., Nguyen T.S., Selvadurai A.P.S., Armand G., Sobolik S.R., Itamura M., Stone C.M., Webb S.W., Rajeb A., Tijani M., Maouche Z., Kobayashi A., Huikami H., Ito A., Sugita Y,. Chijimatsu M., Borgesson L., Hernelind J., Rutqvist J., Tsang C.F., Jussia P. (2005) The FEBEX Benchmark Test: Case definition and comparison of modelling approaches, International Journal of Rock Mechanics and Mining Sciences 42, 611-638.

Atkin R.J., Craine R.E. (1976) Continuum Theories of Mixtures: Basic Theory and Historical Development, Quarterly Journal of Mechanics and Applied Mathematics 29, 209-244.

Bear J. (1972) Dynamics of Fluids in Porous Media, Dover Publications, New York.

Bernaix J. (1969) New Laboratory Methods of Studying the Mechanical Properties of Rocks, International Journal of Rock Mechanics and Mining Science 6, 43-90.

Beauheim R.L., Roberts R.M., Avis J.D. (2014) Hydraulic Testing of Low-Permeability Silurian and Ordovician Strata, Michigan Basin, Southwest Ontario, Journal of Hydrology 509, 163-178.

Biot M.A. (1941) General Theory of Three-Dimensional Consolidation, Journal of Applied Physics 12, 155-164.

Bock H., Dehandschutter B., Martin C.D., Mazurek M., de Haller A., Skoczylas F., Davy C. (2010) Self-Sealing of Fractures in Argillaceous Formations in Context with the Geological Disposal of Radioactive Waste - Review and Synthesis, OECD, NEA, No. 6184, Nuclear Energy Agency, Paris, France.

Bowen R.M. (1976) Theory of Mixtures, in Continuum Physics, Eringen A.C. (ed.), Academic Press, New York-London.

Brace W.F., Walsh J.B., Frangos W.T. (1968) Permeability of Granite under High Pressure, Journal of Geophysical Research 73, 2225-2236.

Coussy O. (1995) Mechanics of Porous Continua, John Wiley \& Sons, Chichester.

David C. (1993) Geometry of Flow Paths for Fluid Transport in Rocks, Journal of Geophysical Research 98, B7, 12267-12278.

David C., Darot M. (1989) Permeability and Conductivity of Sandstones, in Proceedings of the Rock at Great Depth Symposium, Maury V., Fourmaintraux D. (eds), A.A. Balkema, 1: 203-209.

David C., Wong T.-F., Zhu W., Zhang J. (1994) Laboratory Measurement of Compaction-Induced Permeability Change in Porous Rocks: Implications for the Generation and Maintenance of Pore Pressure Excess in the Crust, Pure and Applied Geophysics 143, 425-456.

Davy C., Skoczylas F., Barnichon J.-D., Lebon P. (2007) Permeability of Macro-Cracked Argillite Under Confinement: Gas and Water Testing, Physics and Chemistry of the Earth 32, 667-680.

Davy C., Skoczylas F., Lebon P., Dubois T. (2009) Gas Migration Properties through a Bentonite/Argillite Interface, Applied Clay Science 42, 639-648.

Fuenkajorn K., Daemen J.K.K. (1996) Sealing of Boreholes and Underground Excavations, Springer-Verlag, Berlin.

Gens A. (2011) Soil-Environment Interaction in Geotechnical Engineering, Géotechnique 60, 3-74.

Golder Associates (2003) Low Level Waste Geotechnical Feasibility Study - Western Waste Management Facility Bruce Site, Tiverton, Ontario, Report to Ontario Power Generation.

Golfier F., Lasseux D., Quintard M. (2015) Investigation of the permeability of vuggy or fractured porous media from a DarcyBrinkman approach, Computational Geosciences 19, 63-78. 
Green A.E., Naghdi P.M. (1970) The Flow of Fluid through an Elastic Solid, Acta Mechanica 9, 329-340.

Hart D.J., Wang H.F. (1998) Poroelastic Effects During a Laboratory Transient Pore Pressure Test, in Poromechanics, Thimus J.F., Abousleiman Y., Cheng A.H.-D., Coussy O., Detournay E. (eds), Balkema, Rotterdam.

Horseman S.T. (2001) Self-Healing of Fractures in Argillaceous Media from the Geomechanical Point of View, in Self-healing topical session, Proceedings of 11th Clay Club Meeting, Nancy, OECD/NEA, Paris.

Hoteit N., Su K., Tijani M., Shao J.-F. (eds) (2002) Hydromechanical and Thermohydromechanical Behaviour of Deep Argillaceous Rock-Theory and experiments, Proceedings of the International Workshop on Geomechanics, Paris, A.A. Balkema, Lisse, The Netherlands.

Hueckel T., Peano A. (1996) Thermomechanics of Clays and Clay Barriers, Engineering Geology 41, 1-4.

Jannot Y., Lasseux D. (2012) A new quasi-steady method to measure gas permeability of weakly permeable porous media, Review of Scientific Instruments 83, 015113.

Jenner L. (2012) Radial Hydraulic Flow Testing of an Argillaceous Limestone, M.Eng. Thesis, Department of Civil Engineering and Applied Mechanics, McGill University, Montreal.

Joint Review Panel Report (2015) Environmental Assessment Report. Deep Geologic Repository for Low and Intermediate Level Radioactive Waste Project, CEAA Reference No. 17520, Canadian Nuclear Safety Commission, Ottawa.

Kiyama T., Kita H., Ishijima Y., Yanagidani T., Akoi K., Sato T. (1996) Permeability in Anisotropic Granite Under Hydrostatic Compression and Tri-axial Compression Including Post-Failure Regions, Proceedings of the $2^{\text {nd }}$ North American Rock Mechanics Symposium, 1643-1650.

Maßmann J., Uehara A., Rejeb A., Millard A. (2009) Investigation of Desaturation in an Old Tunnel and New Galleries at an Argillaceous Site, Environmental Geology 57, 1337-1345.

Mahyari A.T., Selvadurai A.P.S. (1998) Enhanced Consolidation in Brittle Geomaterials Susceptible to Damage, Mechanics of Cohesive Frictional Materials 3, 291-303.

Mazurek M. (2004) Long-Term Used Nuclear Fuel Waste Management - Geoscientific Review of the Sedimentary Sequence in Southern Ontario, Technical Report TR 04-01, Institute of Geological Sciences, University of Bern, Switzerland.

Mehta P.K., Monteiro P.J.M. (2014) Concrete. Microstructure, Properties and Materials, McGraw-Hill, New York.

NWMO (2011) Geoscientific Verification Plan, Nuclear Waste Management Organization Document, NWMO DGR-TR-2011-38 R000, Toronto, Canada.

OPG (2008) Ontario Power Generation's Deep Geologic Repository for Low and Intermediate Level Waste, Supporting Technical Report: Phase 1-Geosynthesis. OPG 00216-REP-01300-00010-R00.

Pijaudier-Cabot G., Pereira J.-M. (eds) (2013) Geomechanics in $\mathrm{CO}_{2}$ Storage Facilities, John Wiley \& Sons, New Jersey.

Pusch R. (ed.) (1990) Artificial Clay Barriers for High Level Radioactive Waste Repositories, Engineering Geology 28, 231-464.

Selvadurai A.P.S. (1996a) Heat-Induced Moisture Movement in a Clay Barrier I. Experimental Modelling of Borehole Emplacement, Engineering Geology 41, 239-256.

Selvadurai A.P.S. (1996b) Heat-Induced Moisture Movement in a Clay Barrier II. Computational Modelling and Comparison with Experimental Results, Engineering Geology 41, 219-238.
Selvadurai A.P.S. (1996c) Mechanics of Poroelastic Media, Kluwer Academic Publishers, Boston.

Selvadurai A.P.S. (Ed.) (1997) Hydro-Thermo-Mechanics of Engineered Clay Barriers and Geological Barriers, Special Issue of Engineering Geology 47, 311-379.

Selvadurai A.P.S. (2000a) Partial Differential Equations in Mechanics Vol. 1: Fundamentals, Laplace's Equation, the Diffusion Equation, the Wave Equation, Springer-Verlag, Berlin.

Selvadurai A.P.S. (2000b) Partial Differential Equations in Mechanics Vol. 2: The Bi-Harmonic Equation, Poisson's Equation, Springer-Verlag, Berlin.

Selvadurai A.P.S. (2002) Influence of Pressurized Water Influx on the Hygro-Thermal Behaviour of an Engineered Clay Barrier in a Waste Emplacement Borehole, Engineering Geology 64, 157-178.

Selvadurai A.P.S. (2004a) Fluid Intake Cavities in Stratified Porous Media, Journal of Porous Media 7, 165-181.

Selvadurai A.P.S. (2004b) Stationary Damage Modelling of Poroelastic Contact, International Journal of Solids and Structures 41, 2043-2064.

Selvadurai A.P.S. (2006) Gravity-Driven Advective Transport during Deep Geological Disposal of Contaminants, Geophysical Research Letters 33, L08408.

Selvadurai A.P.S. (2007) The Analytical Method in Geomechanics, Applied Mechanics Reviews 60, 87-106.

Selvadurai A.P.S. (2009) Influence of Residual Hydraulic Gradients on Decay Curves for One-Dimensional Hydraulic Pulse Tests, Geophysical Journal International 177, 1357-1365.

Selvadurai A.P.S. (2010) On the Hydraulic Intake Shape Factor for a Circular Opening Located at an Impervious Boundary: Influence of Inclined Stratification, International Journal for Numerical and Analytical Methods in Geomechanics 35, 6, 639-651.

Selvadurai A.P.S. (2012) Fluid Leakage Through Fractures in an Impervious Caprock Embedded between Two Geologic Aquifers, Advances in Water Resources 41, 76-83.

Selvadurai A.P.S. (2013) Caprock Breach: a Potential Threat to Secure Geologic Sequestration of $\mathrm{CO}_{2}$, in Geomechanics in $\mathrm{CO}_{2}$ Storage Facilities, Pijaudier-Cabot G., Pereira J.-M. (eds), John Wiley \& Sons, New Jersey.

Selvadurai A.P.S. (2015a) Thermo-Hydro-Mechanical Behaviour of Poroelastic Media, Chapter 20, in Handbook of Porous Media, Vafai K. (ed.), Taylor and Francis, U.K.

Selvadurai A.P.S. (2015b) Normal stress-induced permeability hysteresis of a fracture in a granite cylinder, Geofluids, Special Issue on Crustal Permeability 15, 37-47.

Selvadurai A.P.S., Carnaffan P. (1997) A Transient Pressure Pulse Technique for the Measurement of Permeability of a Cement Grout, Canadian Journal of Civil Engineering 24, 489-502.

Selvadurai A.P.S., Głowacki A. (2008) Evolution of Permeability Hysteresis of Indiana Limestone during Isotropic Compression, Ground Water 46, 113-119.

Selvadurai A.P.S., Ichikawa Y. (2013) Some Aspects of AirEntrainment on Decay Rates in Hydraulic Pulse Tests, Engineering Geology 165, 38-45.

Selvadurai A.P.S., Jenner L. (2012) Radial Flow Permeability Testing of an Argillaceous Limestone, Ground Water 51, 1, 100-107.

Selvadurai A.P.S., Najari M. (2013) On the Interpretation of Hydraulic Pulse Tests on Rock Specimens, Advances in Water Resources 53, 139-149. 
Selvadurai A.P.S., Najari M. (2015) Laboratory-Scale Hydraulic Pulse Testing: Influence of Air Fraction in Cavity on Estimation of Permeability, Géotechnique 65, 2, 126-134.

Selvadurai A.P.S., Nguyen T.S. (1997) Scoping Analyses of the Coupled Thermal-Hydrological-Mechanical Behaviour of the Rock Mass around a Nuclear Fuel Waste Repository, Engineering Geology 47, 379-400.

Selvadurai A.P.S., Selvadurai P.A. (2010) Surface Permeability Tests: Experiments and Modelling for Estimating Effective Permeability, Proceedings of the Royal Society, Mathematical and Physical Sciences Series A 466, 2122, 2819-2846.

Selvadurai A.P.S., Shirazi A. (2004) Mandel-Cryer Effects in Fluid Inclusions in Damage-Susceptible Poroelastic Geologic Media, Computers and Geotechnics 31, 285-300.

Selvadurai A.P.S., Singh B.M. (1984) On the expansion of a pennyshaped crack by a rigid circular disc inclusion, International Journal of Fracture 25, 69-77.

Selvadurai A.P.S., Singh B.M. (1985) The annular crack problem for an isotropic elastic solid, Quarterly Journal of Mechanics and Applied Mathematics 38, 233-243.

Selvadurai A.P.S., Boulon M.J., Nguyen T.S. (2005) The Permeability of an Intact Granite, Pure and Applied Geophysics 162, 373-407.

Selvadurai A.P.S., Letendre A., Hekimi B. (2011) Axial Flow Hydraulic Pulse Testing of an Argillaceous Limestone, Environmental Earth Sciences 64, 8, 2047-2058.

Selvadurai P.A., Selvadurai A.P.S. (2014) On the Effective Permeability of a Heterogeneous Porous Medium: the Role of the Geometric Mean, Philosophical Magazine 94, 2318-2338.

Shiping L., Yushou L., Yi L., Zhenye W., Gang Z. (1994) Permeability-Strain Equations Corresponding to the Complete Stress-Strain Path of Yinzhuang Sandstone, International Journal of Rock Mechanics and Mining Sciences and Geomechanics Abstracts 31, 383-391.
Sneddon I.N. (1951) Fourier Transforms, McGraw-Hill, New York.

Terzaghi K. (1923) Die Berechnung der Durchlassigkeitsziffer des Tones aus Dem Verlauf der Hydrodynamischen Spannungserscheinungen, Akad Wissensch Wien Sitzungsber Mathnaturwissensch Klasse IIa 142, 125-138.

Testa S.M. (1994) Geological Aspects of Hazardous Waste Management, CRC Press, Boca Raton.

Tranter C.J. (1971) Integral Transform in Mathematical Physics, Chapman and Hall, London.

Verruijt A. (2014) Theory and Problems of Poroelasticity, Delft University of Technology, The Netherlands.

Vilks P., Miller N.H. (2007) Evaluation of Experimental Protocols for Characterizing Diffusion in Sedimentary Rocks, Atomic Energy of Canada Limited. Nuclear Waste Management Division Report TR-2007-11. Toronto, Ontario.

Wang H.F. (2000) Theory of Linear Poroelasticity with Applications to Geomechanics and Hydrogeology, Princeton University Press, Princeton.

White F.M. (1986) Fluid Mechanics, McGraw-Hill, New York.

Zoback M.D., Byerlee J.D. (1975) The Effect of Microcrack Dilatancy on the Permeability of Westerly Granite, Journal of Geophysical Research 80, 752-755.

Cite this article as: A.P.S. Selvadurai and M. Najari (2016). Isothermal Permeability of the Argillaceous Cobourg Limestone, Oil Gas Sci. Technol 71, 53. 